\title{
OBSERVING TUMOR VASCULARITY NONINVASIVELY USING MAGNETIC RESONANCE IMAGING
}

\author{
Mark E HaAcke ${ }^{1}$, Gwen Herigault ${ }^{1}$, DAniel Kido ${ }^{2}$, Karen Tong ${ }^{2}$, Andre Obenaus $^{2}$, \\ YINGJIAN YU ${ }^{2}$ AND JUERGEN R REICHENBACH ${ }^{3}$ \\ ${ }^{1}$ The Magnetic Resonance Imaging Institute for Biomedical Research, Detroit, Michigan ${ }^{2}$ Departments of \\ Radiology and Radiation Medicine, Loma Linda University, Loma Linda, CA 92324, ${ }^{3}$ Institute of Diagnostic \\ and Interventional Radiology, Friedrich-Schiller, University, Bachstrasse 18, 07740 Jena, Germany \\ e-mail: nmrimaging@aol.com \\ (Accepted May 15, 2002)
}

\begin{abstract}
A new approach to tumor discrimination using magnetic resonance imaging is reported. The susceptibility difference between venous blood and the surrounding tissue is used to generate contrast. This approach is able to exquisitely reveal small veins even those which are smaller than a voxel. Using this method, it is possible to visualize the primary draining veins within tumors better than contrast enhanced magnetic resonance imaging methods that require a contrast agent or even conventional angiography. The ability to highlight deoxygenated blood may lead to the possibility of differentiating benign from malignant tumors noninvasively.
\end{abstract}

Keywords: brain vasculature, susceptibility weighted images, venous system, tumor identification.

\section{INTRODUCTION}

Categorization of benign from malignant tumors presents a major clinical challenge. There are certain pathophysiolgical parameters such as tissue necrosis, neovascularization, rate of growth and metabolic activity in tumors that are considered in any attempt to make this separation. Neuroimaging has been successfully used to monitor tumor growth and necrosis especially with the use of traditional contrast agents. However, a biopsy is usually needed to determine the pathophysiologic nature of a tumor. Given the discrete nature of biopsy sampling and the heterogeneities present in tumors, other non-invasive means of determining the malignant nature of tumors is highly desirable. Our thrust is to use susceptibility differences between partially deoxygenated blood and surrounding tissue to visualize the draining veins in tumors with high resolution MR images in order to make this determination.

Modern vascular studies of tumors focus predominantly on the arterial input to tissues. The resulting images display information about both size, contour and location of the arteries which is useful for performing clinical biopsies. Traditional contrast agents have also been used to assess the rate of uptake in the tumor and blood volume. Furthermore, many investigators have suggested that oxygen utilization being highest in the most malignant tumors, could also be considered as a key parameter for the determination of tumor malignancy. To date, positron emission tomography (PET) has shown some promising results, however, the required radioactive isotopes and the need for an onsite cyclotron have prevented its wide use.

Alternatively, given the present understanding of blood oxygen level dependent (BOLD) contrast, MR imaging offers a new means by which to monitor oxygen utilization. Furthermore, the current interest in using angiostatin drugs to retard tumor growth (Bergers et al., 1999; Carmeliet et al., 1998) makes visualizing small veins particularly important.

To date, the examination of small veins has only been possible using radiographic techniques on cadaver brains (Salamon and Huang, 1976). However, we have recently developed a magnetic resonance imaging sequence to probe the presence of de-oxygenated blood in small vessels (Reichenbach et al., 1997). Previous studies of functional brain imaging using the blood's oxygen levels as an endogenous contrast agent (Belliveau et al., 1991) have demonstrated the ability of MR gradient echo imaging to detect changes 
in tissue activation (Ogawa et al., 1990). More recently, others have used the BOLD effect to study vascular effects in tumors, proposing either alternate imaging methods sensitive to susceptibility by challenging the tissue with oxygen or carbogen and looking for changes in signal caused by changes in the oxygenation level of the blood in the tumor (McSheehy et al., 1998; Al-Hallaq et al., 1998).

Our method, on the other hand, does not search for changes, but directly scans the brain in its resting state to probe the venous side of the vasculature for any abnormalities in oxygen saturation levels or in the volume of venous blood. These irregularities could serve as biomarkers of local disease and may potentially be used as a means to stage the tumors. The ability to detect these changes is dependent on the local changes in susceptibility and hence we refer to the method as susceptibility weighted imaging or SWI.

\section{METHODS}

\section{THEORETICAL CONSIDERATIONS}

We base the design of this sequence on the BOLD signal concept (Ogawa et al., 1990). Since the local magnetic field in and around blood vessels depends on the oxygen saturation in the blood, the choice of echo time can be made to reveal dramatic signal cancellation caused by venous blood. Even vessels which are a fraction of a voxel in size will be visible due to these phenomena. Signal cancellations from the blood (the intravascular contribution) and background tissue occur when a blood vessel is smaller than a voxel. The magnetic fields outside the blood vessel also cause signal loss in the tissue (leading to the extravascular contribution). These two effects make it possible to visualize vessels only a few hundred microns in size within voxels on the order of $1 \mathrm{~mm}^{3}$. Tissue with abnormally high venous blood volume or low levels of oxygen will be dramatically highlighted due to the signal losses just described.

A complex MR image can be presented as a magnitude image and a phase image. To understand the effects on the image caused by the local magnetic fields associated with the blood, it is first necessary to understand the role that phase plays in determining the MR signal. The spin phase, $\varphi$, develops according to the simple linear formula

$$
\varphi=-\gamma \Delta \mathrm{BT}_{\mathrm{E}}
$$

where $\gamma$ is the magnetogyric ratio of protons, $\Delta \mathrm{B}$ is the field difference between blood and the surrounding tissue, and $T_{E}$ is the time at which the data are sampled after the excitatory radiofrequency pulse is applied. For a long, cylindrical-like vessel parallel to the main field, $\Delta \mathrm{B}$ can be written as

$$
\Delta \mathrm{B}=4 \pi \chi_{\mathrm{do}} \mathrm{B}_{0}(1-\mathrm{Y}) \mathrm{Hct} / 3
$$

where $\chi_{\mathrm{do}}=0.18 \times 10^{-6}$ is the susceptibility change between de-oxygenated and oxygenated blood (Weisskoff and Kiihne, 1992), $B_{0}$ is the main static field strength, $\mathrm{Y}$ is the fractional oxygen saturation of the blood in the vessel, and Hct is the fractional hematocrit. Taking $\mathrm{B}_{0}=1.5 \mathrm{~T}, \mathrm{Y}=0.55, \quad \mathrm{Hct}=0.45, \quad$ and $\gamma=2 \pi(43.58) \mathrm{MHz} / \mathrm{T}$ yields the approximate relation

$$
\varphi=-20 \pi \mathrm{T}_{\mathrm{E}}
$$

where $\varphi$ is in radians when TE is given in milliseconds. For higher field strengths, such as $3 \mathrm{~T}$, the echo time can be reduced (halved) and the same effect will be seen. This makes it possible to image with higher resolution in the same imaging time or to reduce the imaging time. Since the SNR is higher as the field strength increases (double at $3 \mathrm{~T}$ ), image quality will also improve (Reichenbach et al., 2000).

When $\mathrm{T}_{\mathrm{E}}=50 \mathrm{~ms}$ and $\varphi=-\pi$, the signal of the venous blood is predicted to oppose that of the background tissue and leads to maximum signal cancellation. Vessels at an arbitrary angle $\vartheta$ to the main field develop an intravascular field proportional to $3 \cos ^{2} \vartheta-1$ as well as an extravascular field which varies as $\sin ^{2} \vartheta \cos 2 \phi / \mathrm{r}^{2}$ (where $\phi$ is the angle between the projection of the B-field and the position vector onto a plane perpendicular to the long axis of the vessel) and which also leads to signal cancellation. Hence, the minimum $T_{E}$ at which maximum signal loss is expected is $50 \mathrm{~ms}$ for the values quoted above and for a vessel parallel to the main field. On the other hand, the intravascular effect vanishes at the magic angle $\vartheta=54.7^{\circ}$ and signal loss will now be exclusively extravascular. By $\vartheta=90^{\circ}$, extravascular cancellations are now at a maximum, and from the intravascular contributions, there can once again be complete cancellation of signal for $\mathrm{T}_{\mathrm{E}}=100 \mathrm{~ms}$.

For oxygen deprived tissue, as $\mathrm{Y}$ decreases, 1-Y increases, and the extravascular signal loss increases because the dephasing from the background field increases. Alternatively, if, in tumors, venous cerebral blood volume increases, the intravascular cancellation increases. In both instances, the diseased tissue will appear darker than healthy tissue. In the former case, the signal loss occurs at shorter echo times, creating the impression of a shorter T2*. It is these mechanisms of signal loss that are used to 
create the enhanced venous functional images (Reichenbach et al., 1997, 1998, 2000).

\section{CLINICAL IMAGING PARAMETERS}

In order to create these "venographic" or "susceptibility weighted" images, a high resolution, fully velocity compensated, 3D, gradient echo, sequence is used (Reichenbach et al., 1997). A repeat time of $60 \mathrm{~ms}$ is used for 192 phase encoding steps and 32 to 64 slices, yielding an imaging time of 6.4 to $12.8 \mathrm{~min}$. In-plane resolution was $0.5 \times 1.0 \mathrm{~mm}^{2}$, while slice thickness was $1 \mathrm{~mm}$ or $2 \mathrm{~mm}$. An MR angiographic sequence was also used for one subject in the presence of a contrast agent with a resolution of $0.5 \times 1.0 \times 1.0 \mathrm{~mm}^{3}$. Informed consent was obtained on all volunteers.

\section{VALIDATION IN AN ANIMAL TUMOR MODEL}

A series of animals were injected with a human derived glioma cell line to study the progression and growth of this tumor type. We applied susceptibility weighted imaging (see above) to determine the extent of the vascularity of these tumors. Briefly, animals $(n=3)$ were implanted with 40,000 CNS-1 glioma cells and the tumor was allowed to grow over the course of 16 days. Due to the aggressive nature of this tumor type animals were often incapable of full locomoter activity and the experiment was terminated. A gradient echo sequence was modified on a Bruker 4.7 T microimager (Bruker Instruments, Billerica, MA) located in the Department of Radiation Medicine, Loma Linda University. All animals were imaged during the course of the 16 day tumor growth and the following imaging parameters were used. A 3D gradient echo sequence with full flow compensation in $\mathrm{read} / \mathrm{phase} / \mathrm{slice}$ select directions with a TR/TE: $54 / 30 \mathrm{~ms}$, FA: 25 , in plane resolution: $0.09375 \times 0.09375 \times 0.5 \mathrm{~mm}^{3}$ was utilized. Total imaging time was $11 \mathrm{~min}$. Gadolinium was injected into the tail vein of the rat $(0.5 \mathrm{ml}$ over $5 \mathrm{~min})$ as a traditional contrast agent. This allowed us to compare the growth.

All of the data was post-processed and the phase image was filtered with $32 \times 32$ filter size using in-house software. No post processing was applied to the magnitude images.

\section{RESULTS}

The SWI images were processed as described previously (Reichenbach et al., 1997) to give a minimum intensity projection image over several adjacent slices. For the angiographic images, a maximum intensity projection was performed. An example of each is shown in Fig. 1 for a volunteer. The images (Fig. 1a) demonstrate exquisite detail for the small deep white matter veins in the brain while showing no arteries and compare well with the known venous structure from radiographic studies of brain sections (Reichenbach et al., 1997). The corresponding contrast enhanced MR angiogram (Fig. 1b) fails to show all the small veins (although the larger vessels are present). A high resolution example of SWI acquired at $3 \mathrm{~T}$ (Fig. 2) demonstrates the very small vessels visible at a shorter echo time of $20 \mathrm{~ms}$ (this is equivalent to the $40 \mathrm{~ms}$ TE time for $1.5 \mathrm{~T}$ as described in the materials and methods section).

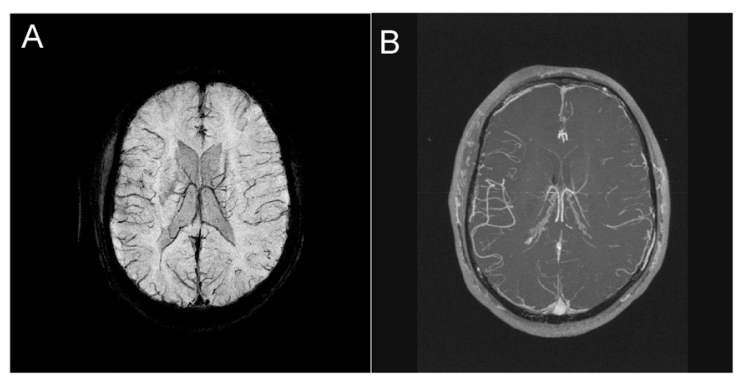

Fig. 1. Projection images over $2 \mathrm{~cm}$ of the vasculature in the central part of the brain. A) This image was acquired using the proposed venographic technique to show dark veins but not affect the arterial signal. B) This image was collected with a contrast agent to enhance the signal from the blood. Since the brain parenchyma also enhances, it is difficult to see the small vessels and small veins in particular. Mostly arteries and large veins are visible in the contrast enhanced image.

Conventional invasive angiography also fails to show these veins. Our method offers the ability to image otherwise occult venous lesions (Lee et al., 1999). Using this approach on a patient with a cavernoma (Fig. 3), the venographic images (Fig. 3b) reveal not only the same major vessels shown in the contrast enhanced case (Fig. 3a) but also an adjacent region of tissue with a significantly reduced signal intensity. As mentioned previously, this signal loss is caused either by a reduction of local oxygen saturation or an increase in local venous blood volume. 


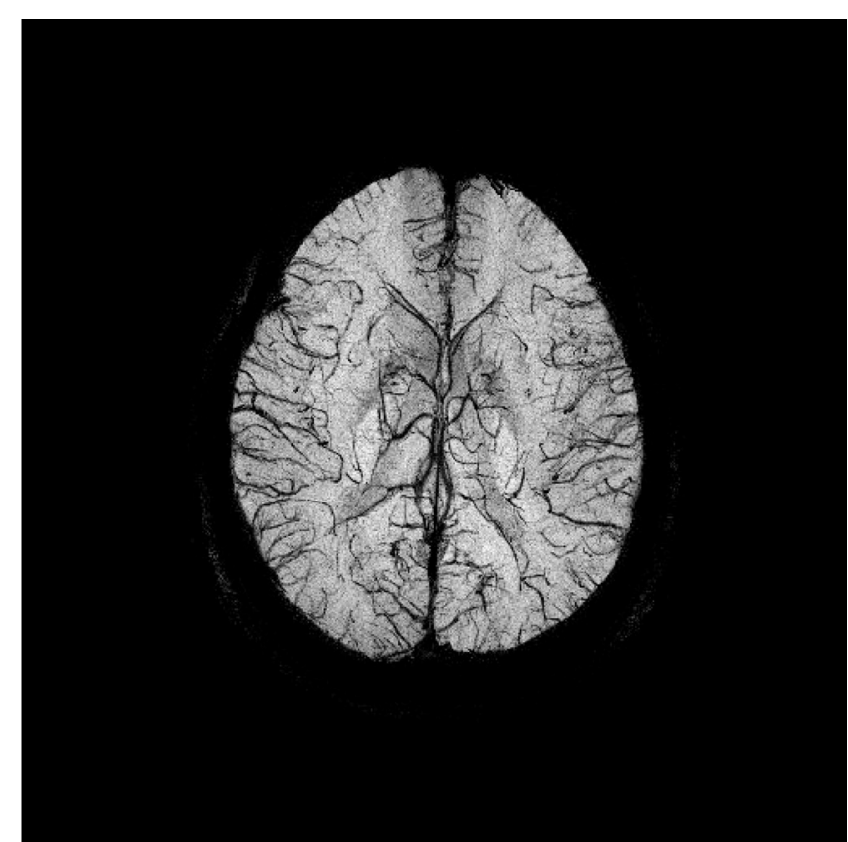

Fig. 2. An SWI image processed from $T E=20 \mathrm{~ms}$ data at 3 T. (Data collected at the University of North Carolina by Gwen Herigault. Sequence modifications from $1.5 T$ to 3 T performed by Weili Lin and Hongyu An.)

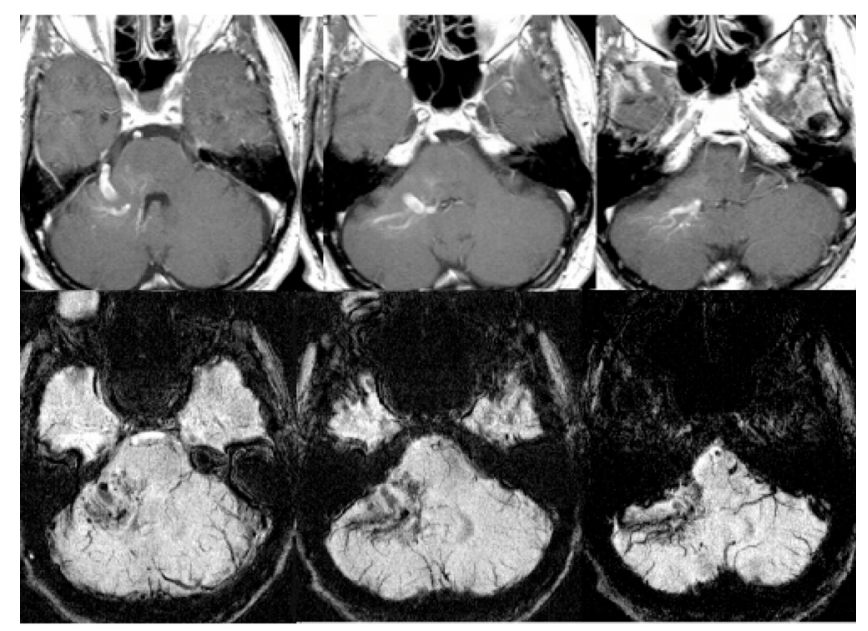

Fig. 3. The top row of images represents three transverse slices from a T1 weighted scan through the cerebellum after the injection of a contrast agent to highlight the abnormal blood vessels. The bottom row is the same set of slices imaged with the new method proposed here to enhance the venous vasculature. Not only do the same regions of large vessels show, but other changes in the surrounding parenchyma are indicated by the adjacent signal loss which appears only as a mild blush in the post contrast case. (Reprinted with permission from AJNR (12)).
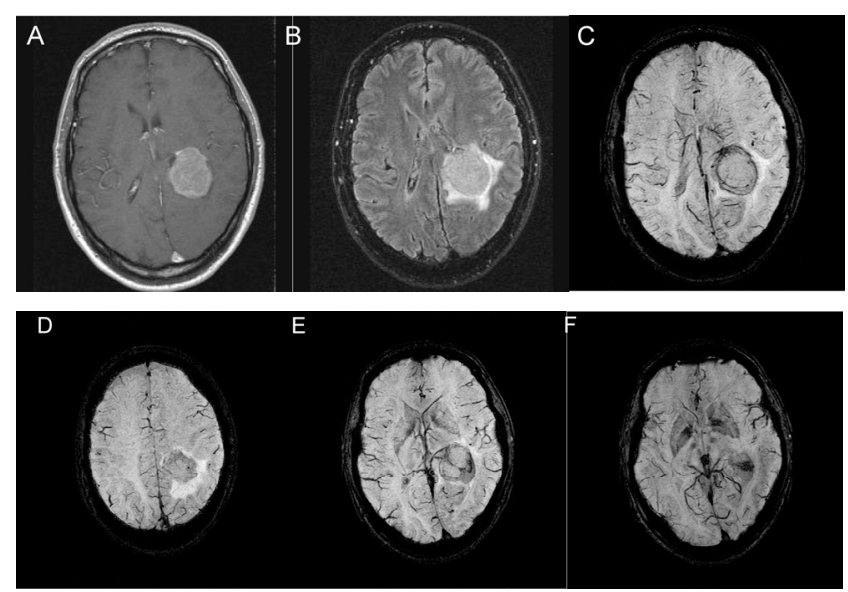

Fig. 4. The visualization of a glioblastoma multiforme with three different imaging methods. A) The first image is a T1 weighted image post-contrast. B) This image is a T2 weighted image. C) The final image is the venographic or susceptibility image. Both the second and third images show the edema surrounding the lesion because of the T2 weighting. D) The visualization of a glioblastoma multiforme has many different appearances with the SWI approach. In D the tumor shows a diffuse darkening indicative of a strong vascular component. E) The dark regions in this image most likely reflect hemorrhage. F) In a slightly different image we can see the vascular nature of tissue surrounding the tumor.

Tumors are usually defined by the extent to which they appear in the contrast enhanced images. On the other hand, our venographic method requires no contrast agent. This is demonstrated in a patient with a glioma multiforme (Fig. 4). The T1 weighted contrast enhanced image (Fig. 4a) shows some peripheral enhancement but does not show the more interior vessels delineated with our approach. Regions of edema are shown in the T2 weighted image but little differentiating contrast within the tumor exists in either the T1 weighted or T2 weighted images. The SWI image (Fig. 4c) clearly shows large veins on the periphery of the tumor which correlate well with the brighter regions in the contrast enhanced image. The next 3 images are contiguous 4 $\mathrm{mm}$ slices in the tumor. They clearly show different contrasts and Fig. 4e and 4f show dark areas that are likely hemorrhage. The darker boundary around the periphery of the tumor in Fig. $4 d$ is suggestive of an increased local venous blood volume content or an increase in the local deoxyhemoglobin in that area. In either case, it appears to have a high vascular activity in this area perhaps suggestive that this could represent the active part of the tumor. 


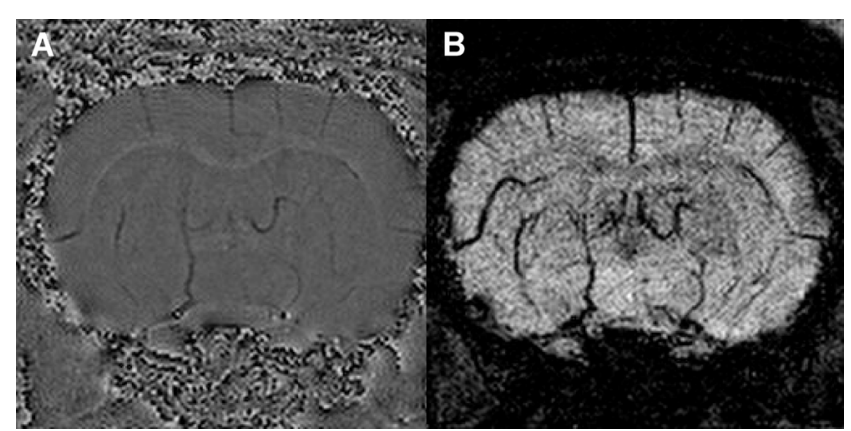

Fig. 5. A phase image (A) and the resulting SWI processed magnitude image (B) of a normal rat brain. The major veins in this area of the rat are demonstrated. The imaging parameters were: $F O V=30 * 30 * 30 \mathrm{~mm}^{3}$,

TE/TR/Flip Angle $=40 \mathrm{msec} / 65 \mathrm{msec} / 30$, matrix $=256 X 256 X 64$ and $N E X=2$.

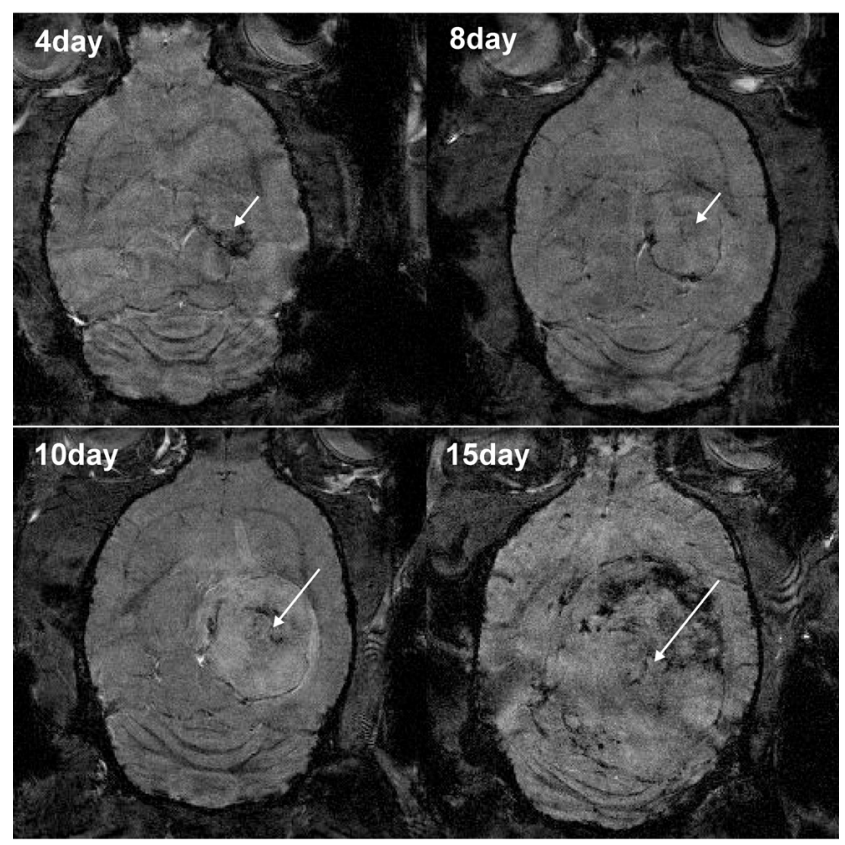

Fig. 6. Glioma tumor progression in an animal model. This figure illustrates the rapid and aggressive growth of this tumor in the rat brain. The tumor appears to grow exponentially over the course of 15 days until almost all of the cerebral cortex and diencephalon are obliterated. The arrow indicates the center of the tumor in these non-contrast susceptibility images.

We have generally seen much better visualization of veins and tissue containing a large volume of venous blood with this approach. Not only are peripheral vessels shown but also more central vessels and regions of signal loss not apparent with other methods such as contrast enhanced imaging or angiographic imaging. The SWI approach can be used to highlight the venous structures (Fig. 5). The major veins in the rat brain are clearly visible.
We have extended these findings in patients to a tumor model in the rodent. Implantation of glioma cells into the cortex of the rat brain leads to rapid growth during the subsequent 16 days. The tumor growth can be readily visualized using our novel imaging method (Fig. 6). A unique capability of the SWI imaging technique is that the tumor can be visualized without traditional contrast agents. As shown in Fig. 6 the growth of the tumor is clearly visible at 4 days post implantation using conventional MRI

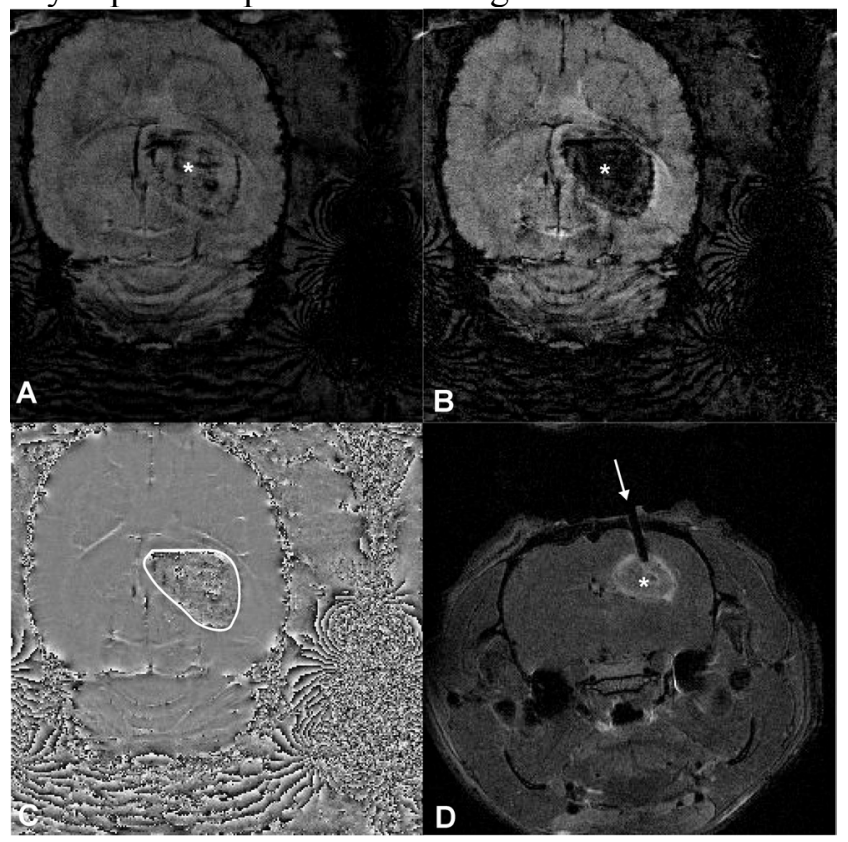

Fig. 7. Multiple imaging methods outline a CNS-1 glioma tumor in the rat brain. A) This is a susceptibility weighted image without contrast that defines the border of the tumor (asterisk indicates the center of the tumor). B) Injection of Gadolinium dramatically demarcates the boundary of the tumor at 10 days. C) The phase encoded image demonstrates a dark punctate appearance within the region demarcated by the tumor (white line). This hypothesis will await histological verification. D) Traditional T1 weighted imaging with contrast enhancement reveals a well demarcated tumor within the brain of the rat. The arrow indicates the cannula tract where the glioma cells were injected.

methods but could be seen in SWI images as early as 2 days. Contrast injection helped to further delineate the tumor boundaries but was found not to be integral for detection of the tumor. We then compared susceptibility weighted imaging with traditional $\mathrm{T} 1$ weighted imaging with contrast (Fig. 7). In Fig. 7A and $\mathrm{B}$ we compare the pre-contrast and post-contrast images of a glioma tumor. As can be seen the susceptibility dramatically increases following the addition of Gadolinium contrast. However, it is 
important to note that the boundaries of the tumor are still apparent in the pre-contrast image (Fig. 7A).

The phase reconstructed image (Fig. 7C) is very provocative as the dark punctate appearance within the tumor is somewhat suggestive of venous structures that may course through the tumor. Histological verification will be required to validate this finding. Finally, a T1 weighted image after contrast is shown for comparison with the susceptibility weighted images and demonstrates that susceptibility weighted imaging can define the border of the tumor more clearly (Fig. 7D). In summary, in an animal model of tumor growth, susceptibility weighted imaging can differentiate the vascularity of the tumor more clearly than conventional imaging sequences.

\section{DISCUSSION}

There are currently still some problems with this method in that hemorrhage can mimic the signal cancellation effect between veins, and background tissue and air/tissue interfaces can lead to signal loss due to the local field variations their presence creates. The former has the potential of being recognized if the same sequence is run after injection of a contrast agent (Lin et al., 1999). In that case, blood vessels will change their signal intensity but regions of clot will not. The latter restricts the region of coverage to the central and upper regions of the brain.

The choice of resolution for these venographic scans will depend on the vessel size of interest and available signal-to-noise (Reichenbach et al., 1998). For vessels of diameter greater than roughly $200 \mu$ and using the imaging parameters described above, good cancellation occurs (Lee et al., 1999).). The previous images were acquired at $1.5 \mathrm{~T}$. Imaging at higher fields offer better signal-to-noise and may offer even better resolution and, therefore, the ability to image smaller vessels yet. There are some indications that imaging at very long echo times (Haacke et al., 1995, 1997) can reveal venules as well. With this capability, it may be viable to predict where activation occurs in functional brain imaging.

Usually arteries appear brighter than veins in these images. This can be understood from the fact that the T2* of arterial blood is roughly $200 \mathrm{~ms}$ while that for venous blood is about $100 \mathrm{~ms}$ or less depending on the oxygen saturation. Since the T2 of surrounding tissue is also $100 \mathrm{~ms}$, the veins normally would appear similar in signal intensity or darker than the surrounding parenchyma because the $\mathrm{T} 1$ weighted sequence also suppresses the signal of the blood relative to the gray matter and T1 for blood is several hundred milliseconds longer than that for gray matter at $1.5 \mathrm{~T}$. However, the arterial signal for longer TEs will be larger than that of the venous blood and it will tend to blend in with the parenchymal signal (depending on the amount of T1 weighting that is present). The phase of the arteries is not affected by the long TE and hence the arteries stay bright and in-phase with the surrounding tissue. For this reason, this behavior of bright arteries and dark veins would still be expected even after injection of a low susceptibility, T1 reducing contrast agent despite the potential increased signal in both arteries and veins since the venous signal still cancels with that of the surrounding brain tissue (Cheng et al., 2001). Hence, we create a natural separation of arteries and veins (Reichenbach et al., 1998). On some occasions, if the flow compensation is not sufficient and fast flow takes place in vessels like the middle cerebral arteries, then the arteries may also appear dark. However, these intrusions are usually easily identified and have not to date created a problem interpreting the images.

The SWI technique also can be easily applied to the studying various disease processes within the nervous system of the rodent. In our work with a tumor model, SWI was able to differentiate tumor boundaries more clearly than conventional contrastenhanced T1 weighted imaging. Using the natural susceptibility of deoxygenated blood we can demonstrate the utility and improved imaging capabilities of SWI.

\section{CONCLUSION}

The ability to visualize the venous system in vivo has significant ramifications for understanding brain function in diseased states. It offers the ability to image tumors without a contrast agent (Jürgen et al., 2001; Schad, 2001), it demonstrates the vascular nature of the lesion, and it indicates that the visible veins have lower oxygen saturation than the incoming arteries. In the future, it may be possible to actually quantify the oxygen saturation of the venous blood using similar methods. The major benefit of this research would be to make it possible to identify tissue at risk where either oxygen saturation has decreased or venous blood volume has increased. In that case, it would be viable to follow patients with tumors as they were being treated and to better diagnose brain tissue damage in general. 


\section{ACKNOWLEDGEMENTS}

Supported in part by the National Institutes of Health via grant number HL62983 and a grant from Siemens Medical Systems, Inc.

\section{REFERENCES}

Al-Hallaq HA, River JN, Zamora M, Oikawa H, Karczmar GS (1998). Correlation of magnetic resonance and oxygen microelectrode measurements of carbogeninduced changes in tumor oxygenation. Int $\mathrm{J}$ Radiat Oncol Biol Phys 41:151-60.

Belliveau JW et al. (1991). Functional mapping of the human visual cortex by magnetic resonance imaging. Science 254:716-9.

Bergers G, Javaherian K, Lo KM, Folkman J, Hanahan D (1999). Effects of angiogenesis inhibitors on multistage carcinogenesis in mice. Science 284:80812.

Carmeliet P et al. (1998). Role of HIF-1alpha in hypoxiamediated apoptosis, cell proliferation and tumour angiogenesis. Nature 394:485-90.

Cheng YCN, Cheng YN, Haacke EM (2001). Predicting BOLD Signal Changes as a Function of Blood Volume Fraction and Resolution. NMR in Biomedicine 14:468-77.

Haacke EM, et al. (1997). In vivo measurement of blood oxygen saturation using magnetic resonance imaging: A direct validation of the blood oxygen leveldependent concept in functional brain imaging. Human Brain Mapping 5:341-6.

Haacke EM, Lai S, Yablonskiy D, Lin W (1995). In vivo validation of the BOLD mechanism: a review of signal changes in gradient echo functional MRI in the presence of flow. International Journal of Imaging Syst Technology 6:153-63.
Jürgen R, Reichenbach JR, Haacke EM (2001). Highresolution BOLD venographic imaging: a window into brain function. NMR in Biomedicine 14:453-67.

Lee BC, et al. (1999). MR high-resolution bold oxygenation level-dependent venography of occult (low-flow) vascular leisons. Am J Neuroradiol 20:1239-42.

Lin W, et al. (1999). Improving high-resolution MR BOLD venographic imaging using a $\mathrm{T} 1$ reducing contrast agent. JMRI 10:118-23.

McSheehy MJ et al. (1998). Carbogen breathing increases 5-fluorouracil uptake and cytotoxicity in hypoxic murine RIF-1 tumors: a magnetic resonance study in vivo. Cancer Research 58:1185-94.

Ogawa S, Lee T, Kay AR, Tank DW (1990). Brain magnetic resonance imaging with contrast dependent on blood oxygenation. Proc Natl Acad Sci 87:9868-72.

Reichenbach JR et al. (1998). High-resolution venography of the brain using magnetic resonance imaging. Magnetic Resonance Materials in Biology, Physics and Medicine 6:62-9.

Reichenbach JR, Barth M, Haacke EM, Klarhöfer M, Kaiser WA, Moser E (2000). High-Resolution MR Venography at 3 Tesla. JCAT 24:949-57.

Reichenbach JR, Venkatesan R, Schillinger DJ, Kido DK, Haacke EM (1997). Small vessels in the human brain: MR venography with deoxyhemoglobin as an intrinsic contrast agent. Radiology 204:272-7.

Salamon G, Huang YP (1976). Radiologic anatomy of the brain. Berlin: Springer-Verlag.

Schad L (2001). Improved target volume characterization in stereotactic treatment planning of brain lesions by using high-resolution BOLD MR-venography. NMR in Biomedicine 14:478-83.

Weisskoff RM, Kiihne S (1992). MRI susceptometry: image-based measurement of absolute susceptibility of MR contrast agents and human blood. Magn Reson in Med 24:375-83. 